\title{
Idiopathic macular holes and direction of vitreomacular traction: structural changes and surgical outcomes
}

\author{
C-Y Tsai ${ }^{1}$, Y-T Hsieh ${ }^{1}$, T-T Lai ${ }^{1}$ and C-M Yang ${ }^{1,2}$
}

\begin{abstract}
Purpose To compare the structural changes, clinical course, and treatment outcomes of

Eye (2017) 31, 1689-1696; doi:10.1038/eye.2017.141; published online 21 July 2017
\end{abstract} vertical and horizontal vitreomacular traction (VMT) induced impending macular holes (IMHs) and full-thickness macular holes (FTMHs).

Methods In this retrospective study, 23 and 32 cases of IMHs and FTMHs, respectively, were analyzed. The IMH cases were divided into two subgroups: IMH with and without foveal detachment. Vitreofoveal traction angles (TAs) between the inner retinal surface and posterior hyaloid were measured from horizontal and vertical optical coherence tomography (OCT) images by using the trigonometric function (the angle equals the arctangent of the height over the base) after adjustments for magnification factors. The largest angle was defined as the vitreomacular TA for the examined case. The critical angle - the TA differentiating cases with (vertical traction) or without (horizontal traction) foveal detachment (vertical traction)—was determined using regression analysis. Pretreatment and posttreatment OCT images, clinical courses, and treatment outcomes were compared between the two groups.

Results The critical angle was $27.2^{\circ}$. Cases of vertical traction had higher foveal height in the IMH group and wider bases in the FTMH group ( $P<0.05$ respectively). IMHs with vertical traction had greater VM attachment than those with horizontal traction. In the FTMH group, postoperative visual improvement was lower $(P=0.002)$; in the vertical traction group, inner segment:outer segment defects persisted longer $(P=0.02)$. Conclusions The critical angle separating vertical from horizontal traction was $27.2^{\circ}$. Vertical VMT results in greater foveal structural changes in IMHs and possibly less favorable surgical outcomes in FTMHs.
Introduction

The most common mechanism for the development of idiopathic full-thickness macular holes (FTMHs) involves vitreomacular traction (VMT) ${ }^{1-4}$ This traction was caused by vitreofoveal adhesion with vitreomacular (VM) separation during the aging of the vitreous. ${ }^{5,6}$ VM separation is typically shallow, with the posterior hyaloid $(\mathrm{PH})$ remaining in contact with the disc and most of the retina. Recent studies have reported that the earliest changes in the fovea involve inner foveal cysts, with or without subtle changes in the foveal outer segment (OS). ${ }^{7-12}$ However, other investigators have noted that in some cases, the earliest changes may be the development of a small foveal detachment with or without inner foveal cysts, instead of inner cysts alone. ${ }^{13-16}$ A recent study suggested that the direction of the traction angle (TA) determines early structural changes in the fovea. Horizontal traction tends to create inner foveal cysts, whereas foveal detachment is likely to be formed because of more anteriorly and posteriorly oriented traction. ${ }^{17}$ If this theory is correct, some questions arise regarding traction direction and foveal changes being predisposing factors for FTMHs. The actual vectors acting on the foveal area caused by the PH are complex and difficult to determine. We postulated that a greater angle of separation between the $\mathrm{PH}$ and retinal surface indicates a more vertical VMT, and this configuration tends to induce foveal detachment before causing FTMHs. Conversely, we considered that FTMHs developed from vitreofoveal adhesion with shallow VM separation may have structural changes and outcomes different from those developed from a significantly larger TA. In this retrospective
${ }^{1}$ Department of Ophthalmology, National Taiwan University Hospital, Taipei, Taiwan, Republic of China

${ }^{2}$ Medical College, National Taiwan University, Taipei, Taiwan, Republic of China

Correspondence:

C-M Yang, Department of Ophthalmology, National

Taiwan University Hospital, 12F, No. 7, Chung-Shan S. Road, Taipei 100, Taiwan, Republic of China Tel: +886 223123456 ext 65187;

Fax: +886 223934420. E-mail: chungmay@ntu.edu. tw

Received: 5 March 2017 Accepted in revised form: 13 June 2017

Published online: 21 July 2017 
review, we analyzed cases of impending macular holes (IMHs) and early FTMHs. By correlating the prehole conditions with the different angles of separation of VMT, we determined a clinically useful parameter that may distinguish more vertically oriented VMT (indicated by foveal detachment in the IMH stage) from more horizontally oriented VMT (indicated by a lack of foveal detachment). Moreover, we investigated the differences in the clinical courses and surgical prognosis between the two study groups.

\section{Materials and methods}

From January 2011 to December 2014, 55 consecutive patients who received a diagnosis of idiopathic IMH $(n=23)$ or stage 2 FTMH $(n=32)$ in a medical centerNational Taiwan University Hospital were retrospectively enrolled in this study. Patients with other diseases, such as strong myopia, retinal detachment, or age-related macular degeneration, were excluded. The staging of

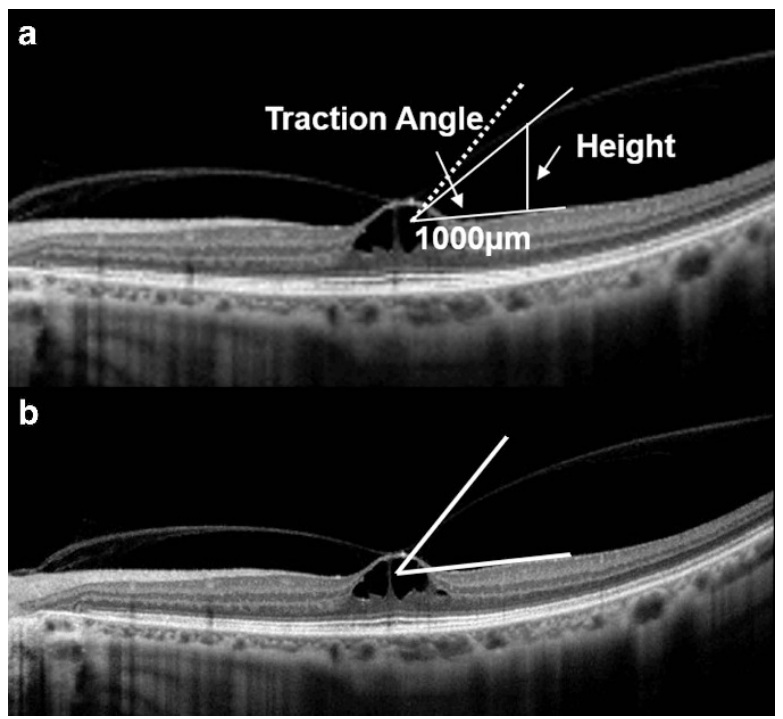

Figure 1 Measurement and calculation of the vitreomacular traction angle (VMTA) in cases of IMHs. (a) Two lines forming the VMTA were drawn first: one along the inner retina and the other along the PH. The actual distance $1000 \mu \mathrm{m}$ from the fovea along the horizontal line was subsequently calculated and marked, after adjustments for magnification factors. The vertical height between the horizontal line (inner retina) and oblique line $(\mathrm{PH})$ at the $1000-\mu \mathrm{m}$ point was measured and adjusted using the magnification factor. The actual angle was calculated by applying trigonometric functions. The actual TA in this particular case was $14.9^{\circ}$ found from arctangent calculations. The angle between the horizontal line and the dot line is CVMTA, which is $27.2^{\circ}$ after calculation. (b) Demonstration of the 'directly measured critical angle'. The angle between the horizontal and oblique lines is $45^{\circ}$ by direct measurement. The image was obtained using the Cirrus HD OCT (Carl Zeiss).
FTMHs was a modification of the classification described by Gass ${ }^{18}$ based on the recent International Vitreomacular Traction Study Group classification for diseases of the VM interface, ${ }^{6}$ and the staging was assisted by optical coherence tomography (OCT). We defined IMHs as focal $(<1500 \mu \mathrm{m})$ VMT causing foveal pseudocysts, foveal detachment, or a combination on an OCT image, and a foveal yellow dot or a ring on a biomicroscopy image. A stage 2 FTMH was defined to have a diameter of $400 \mu \mathrm{m}$ or less and a centrally or eccentrically located opening combined with VM separation and vitreofoveal adhesion. The following demographic and ophthalmic examination data were recorded at baseline: age, sex, history of ophthalmic surgery, follow-up duration, severity of cataracts, and macular appearance obtained from color fundus photographs.

We recorded the morphological changes using OCT, including the size of FTMHs, widest gap (base) of IMHs or FTMHs, height of holes (the distance between the foveal roof and pigment epithelium), length of VM attachment, and presence of foveal detachment or intraretinal cysts in the IMHs. OCT images captured preoperatively or after at least 6 months of follow-up without configuration changes were selected for evaluation and measurement. The length of VM attachment was measured using an electronic caliper in OCT on both horizontal and vertical scans. The greater length was defined as the length of VM attachment for the examined case. Furthermore, treatment methods, pre-operative and postoperative best-corrected visual acuity (BCVA), clinical courses, and detailed postoperative morphological changes on OCT were recorded. All patients received regular follow-ups in our special retinal clinic for more than 1 year. This study was approved by the Institutional Review Board of National Taiwan University Hospital.

\section{Measurement of vitreomacular traction angle}

For each case, we performed OCT using a macular cube (Carl Zeiss Meditec, Inc., Dublin, CA, USA) and crossline (Optovue, Fermont, CA, USA and Heidelberg, Germany) to measure the vitreofoveal TA by using the following methods. Two lines were drawn from the center of the fovea: one along the normal inner retinal surface and the other along the detached $\mathrm{PH}$. In the curved $\mathrm{PH}$, the line was determined by connecting two points: (1) the fovea and (2) the intersection of the $\mathrm{PH}$ and vertical line perpendicular to the inner retina at $1000 \mu \mathrm{m}$ from the fovea. The height of VM separation at $1000 \mu \mathrm{m}$ from the foveal center was measured (Figure 1). All measurements were performed by an ophthalmologist and rechecked by another retinal specialist, and the height was the average of three measurements. Because the OCT images were magnified by different magnification factors in the 
vertical and horizontal directions, we measured the true distance in both directions after adjustments for magnification factors provided by companies that manufactured each OCT machine. We subsequently calculated the actual angle between the inner retinal surface and $\mathrm{PH}$ by using the trigonometric function (the angle equals the arctangent of the height over the base). On each image, two such angles were present; one on each side of the fovea. The largest of the four angles (two from vertical and two from horizontal scans) was defined as the vitreomacular traction angle (VMTA) of the examined case. Three OCT instruments were used for examinations, obtained from Carl Zeiss (Cirrus HD OCT), Optovue OCT, and Heidelberg (Spectralis-OCT).

\section{Determination of critical VMTA}

We hypothesized that a more vertically oriented VMT tends to induce foveal detachment in cases of IMHs, whereas a more horizontally oriented VMT tends to not induce foveal detachment. Therefore, we performed receiver operating characteristic curve analysis to determine the cutoff point of the VMTA-as measured using the methods reported in the previous paragraphwhich was associated with foveal detachment. This cutoff point was called the critical VMTA (CVMTA) and represented the pivotal angle separating horizontal and vertical traction. Vertical traction was defined as the TA exceeding the CVMTA without bending of the vitreous membrane toward the retinal surface; otherwise, the traction was defined as horizontal traction. The phrases 'vertical traction' and 'horizontal traction' were used for classifications in subgroup analysis, as reported in the following section.

\section{IMHs}

For clinical assessment and comparison, the IMH cases were divided into two groups: cases of significant anterior-posterior (AP) traction or vertical traction (group A) and those of tangential or horizontal traction (control A). 'Vertical traction' was defined as follows:

1. OCT images showed that the VMTA exceeded the CVMTA, with no bending of the vitreous membrane toward the retinal surface of that particular angle.

2. If surgery was performed, intraoperative observation during triamcinolone-assisted $\mathrm{PH}$ removal confirmed the separation of the $\mathrm{PH}$ from outside the arcade in the direction suggested by the VMTA in the OCT images.

Those cases with a VMTA of less than the CVMTA served as the control (horizontal traction, control A).
Demographics, basal ophthalmic examination results, clinical courses, and outcomes were compared between the two groups.

For further assessment of the traction effect on FTMHs, we divided the cases of stage 2 FTMHs into FTMHs with vertical traction (group B) and horizontal traction (control B), based on the same criteria as were used for IMHs. Basic information, clinical course, and postoperative macular conditions were compared between the two groups.

\section{Practical consideration of the CVMTA}

To make the CVMTA more applicable for clinical use, we drew it on the OCT images shown on the computer monitor or printouts. The resulting angles obtained on the monitors of the three OCT machines were directly measured and recorded without adjustment for the magnification factors of the machines. We subsequently selected a common and easily identifiable angle close to the three measured figures. This angle was called the 'directly measured critical angle.' Analysis was performed to determine whether the directly measured critical angle can be used to distinguish the traction direction.

\section{Statistics}

The CVMTA was mathematically derived from the receiver operating characteristic curve analysis by using the point on the receiver operating characteristic curve with the lowest value of $\left[(1-\text { sensitivity })^{2}+\right.$ $\left.(1 \text { - specificity })^{2}\right]$ for foveal detachment in cases of IMHs. Student's $t$-test and Fisher's exact tests were used to compare the data between the study and control groups. Statistical significance was set at $P<0.05$ in both tests. SAS 9.4 (SAS Institute Inc., Cary, NC, USA) was used for all statistical analyses.

\section{Results}

The regression analysis of the TA and presence of foveal detachment yielded an actual CVMTA of $27.2^{\circ}$ (specificity $=1.0$ and sensitivity $=1.0$ ). The patients with an actual VMTA exceeding $27.2^{\circ}$ and who fulfilled the other criteria for significant AP traction were defined as the vertical traction group (Figure 2) and those having an actual VMTA of less than $27.2^{\circ}$ were defined as the horizontal traction group (Figure 3). All patients fulfilling criterion 1 for 'significant AP traction' also met criterion 2.

In this study, 55 eyes of 55 patients were examined. Of the 23 eyes with IMHs, 8 were included in group A, and the remaining 15 were included in control A. Among the IMH cases, five and two patients with vertical and horizontal traction developed FTMHs during follow-up. 


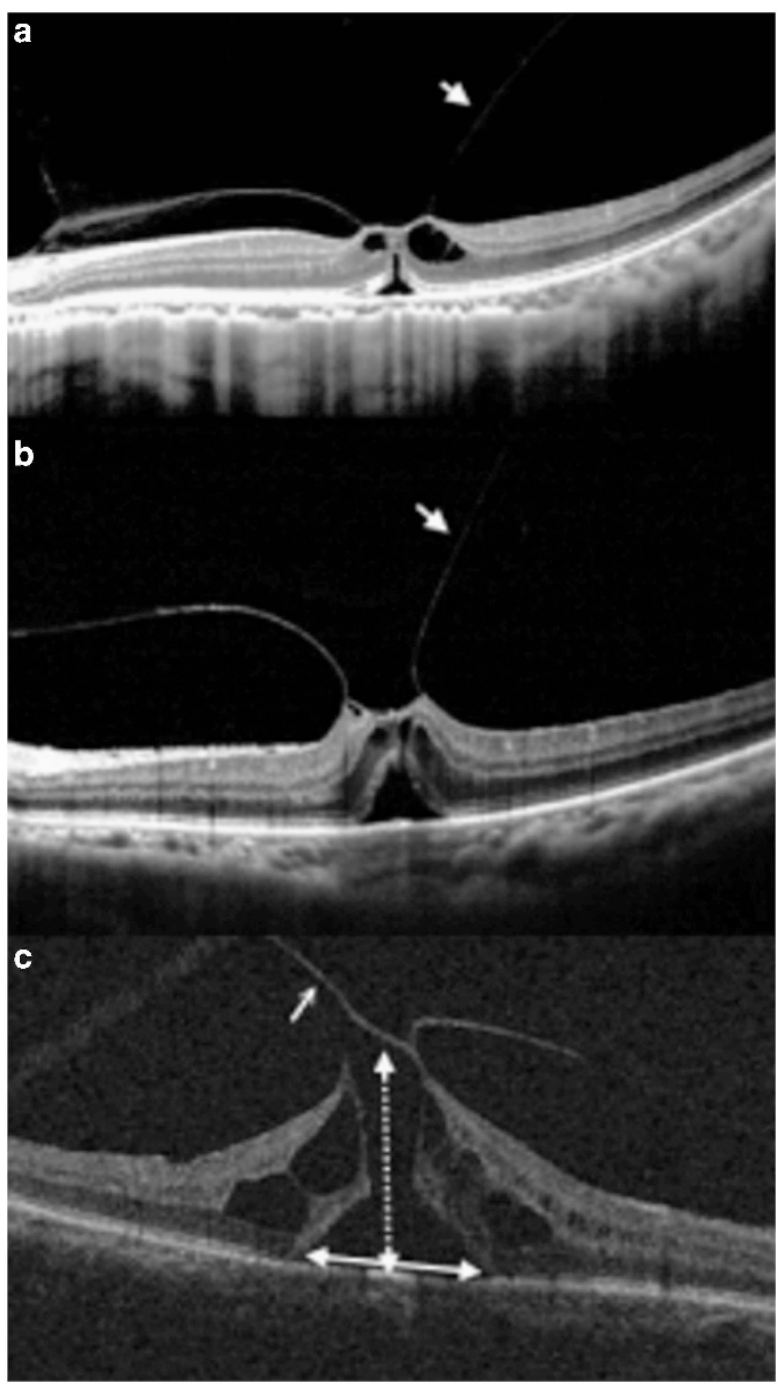

Figure 2 Examples of IMHs and FTMHs with vertical traction. VMT is indicated with arrows. (a) Actual TA by calculation of this IMH was $31.9^{\circ}$. Subretinal fluid and intraretinal cysts were noted in this IMH. (b) Actual TA of this IMH was $42.9^{\circ}$, and subretinal fluid was observed. (c) Actual TA of this FTMH was $44.6^{\circ}$. The wide base is highlighted with a double-headed arrow, and the large height is marked using a double-headed arrow with dotted lines. Image of (a) was obtained using Optovue OCT; images of (b) and (c) using Zeiss Cirrus HD OCT.

Pre-treatment OCT images revealed that group A had a greater average IMH height $(P=0.008)$ and a higher rate of foveal detachment $(P<0.001)$ than did control A. The average length of $\mathrm{VM}$ attachment in group $\mathrm{A}$ was $593.9 \pm 142.3 \mu \mathrm{m}$, which exceeded that in control A $(300 \pm 100.8 \mu \mathrm{m} ; P=0.0014)$. Although the difference was statistically nonsignificant, group A tended to have wider bases and worse BCVA preoperatively than did control A $(P=0.076$ and 0.076; Table 1$)$.

Among the eight patients with vertical traction, five developed FTMHs and three recovered after an

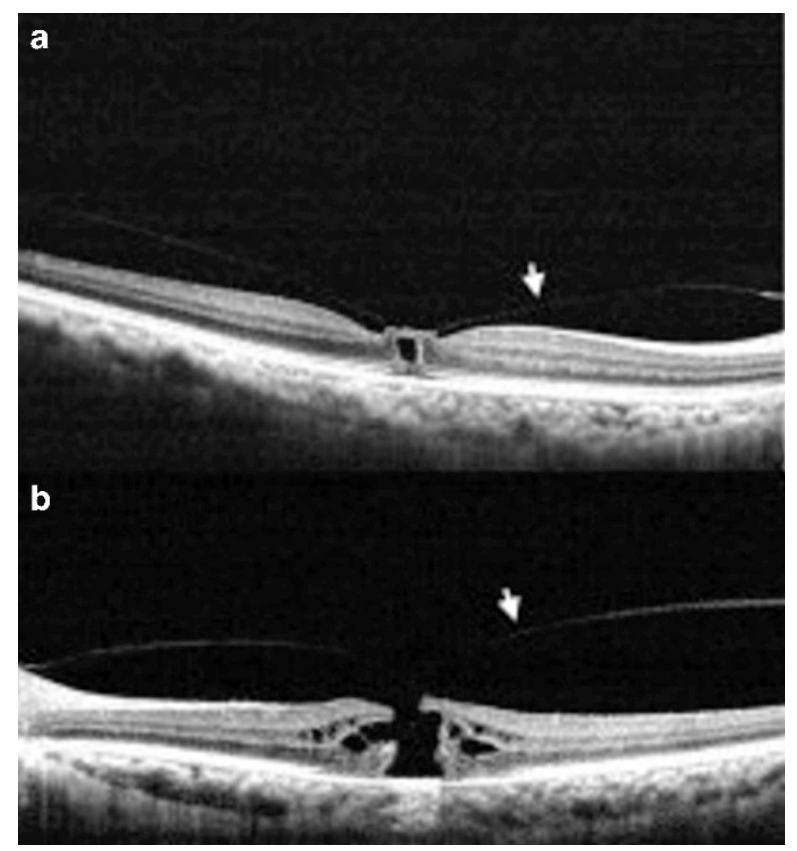

Figure 3 Examples of IMHs and FTMHs with horizontal traction. VMT is indicated with arrows. (a) Actual TA by calculation of this IMH was $2.09^{\circ}$. (b) Actual TA of this FTMH was $16.9^{\circ}$. Both images were obtained using Optovue OCT machine.

intravitreal injection of $\mathrm{C}_{3} \mathrm{~F}_{8}$. Among the five cases that developed FTMHs, the average duration from IMH to FTMH development was 15.7 days (7-30 days). The FTMHs sealed postoperatively in all cases, and a persistent inner segment (IS):OS defect was observed in four out of five cases at a point 1 year postoperation. Conversely, among the 15 patients in control A, 6 received an intravitreal injection of $\mathrm{C}_{3} \mathrm{~F}_{8}$ or $\mathrm{SF}_{6}$, and the holes in 5 sealed postoperatively; one patient developed an FTMH (within 60 days), which sealed after vitrectomy. Among the nine cases without an intravitreal gas injection, one developed an FTMH within 7 days, and the hole sealed after vitrectomy. The remaining eight cases did not receive any treatment during follow-up. One of the eight cases had spontaneous hole closure; IMHs persisted in the remaining seven cases, without significant changes in the VMTA for at least 1 year.

In the FTMH group, we examined 32 eyes of 32 patients, 10 with vertical traction (group B) and 22 with horizontal traction (control B). The IMHs in group B had a significantly greater average height $(P=0.001)$ and wider bases $(P=0.004)$ than did those in control B. However, the IMH size did not significantly vary between the two groups $(P=0.34)$. After surgical treatment, the closure rate was not different between the two groups. However, group B had more cases of IS-OS defects than did control $\mathrm{B}$ at the end of a 1-year follow-up $(P=0.02)$. The pre- 
Table 1 Comparison of the demographics and foveal morphology between impending macular holes (IMHs) with vertical and tangential traction

\begin{tabular}{|c|c|c|c|}
\hline Characteristics & IMHs with vertical traction ${ }^{\mathrm{a}}(\mathrm{n}=8)$ & IMHs with tangential traction ${ }^{\mathrm{b}}(\mathrm{n}=15)$ & P-value \\
\hline Age (years; mean $\pm S D$ ) & $66 \pm 7.35$ & $57.7 \pm 6.7$ & 0.14 \\
\hline \multicolumn{4}{|l|}{ Gender (no./\%) } \\
\hline Male & $3(37.5)$ & $6(40)$ & 1 \\
\hline Female & $5(62.5)$ & $9(60)$ & \\
\hline Spherical equivalent $(\mathrm{SE}$, degree; mean $\pm \mathrm{SD}$ ) & $-1.22 \pm 3.1$ & $-2.63 \pm 3.9$ & 0.39 \\
\hline $\begin{array}{l}\text { History of previous non-vitreoretinal surgery } \\
\text { (no./\%) }\end{array}$ & $3(37.5)$ & $9(60)$ & 0.65 \\
\hline Follow-up time (months; mean $\pm \mathrm{SD}$ ) & $30.9 \pm 18.0$ & $26.1 \pm 26.1$ & 0.50 \\
\hline Pre-operative base of IMH $(\mu \mathrm{m}$; mean \pm SD) & $811.3 \pm 478.2$ & $419.5 .4 \pm 278$ & 0.076 \\
\hline Pre-operative height of IMH $(\mu \mathrm{m}$; mean \pm SD) & $554.7 \pm 284.9$ & $285.6 \pm 107$ & 0.008 \\
\hline $\begin{array}{l}\text { Length of vitreomacular attachment }(\mu \mathrm{m} \text {; } \\
\text { mean } \pm \text { SD) }\end{array}$ & $593.9 \pm 142.3$ & $300 \pm 100.8$ & 0.0014 \\
\hline Presence of foveal detachment (no./\%) & $8(100)$ & $0(0)$ & $<0.001$ \\
\hline BCVA (logMAR; mean $\pm S D)$ & $1.3 \pm 0.3$ & $0.51 \pm 0.29$ & 0.076 \\
\hline
\end{tabular}

Abbreviations: BCVA, best-corrected visual acuity; IMH, impending macular hole. ${ }^{a}$ Vertical traction $=$ The actual traction angle more than the critical vitreomacular traction angle (CVMTA) (27.2 degrees). ${ }^{\mathrm{b}}$ Tangential traction $=$ The actual traction angle less than CVMTA.

Table 2 Comparison of the demographics and foveal morphology between full-thickness macular holes (FTMHs) with vertical and tangential traction.

\begin{tabular}{|c|c|c|c|}
\hline Characteristics & $\begin{array}{l}\text { FTMHs with vertical traction }{ }^{\mathrm{a}} \\
\qquad(\mathrm{n}=10)\end{array}$ & $\begin{array}{l}\text { FTMHs with tangential traction }{ }^{\mathrm{b}} \\
\qquad(\mathrm{n}=22)\end{array}$ & P-value \\
\hline Age (years; mean $\pm S D$ ) & $57.0 \pm 20.7$ & $59.7 \pm 7.16$ & 0.76 \\
\hline $\begin{array}{l}\text { Gender }(\text { no. } / \%) \\
\text { Male } \\
\text { Female }\end{array}$ & $\begin{array}{l}4(40) \\
6(60)\end{array}$ & $\begin{array}{c}3(13.6) \\
19(86.3)\end{array}$ & 0.17 \\
\hline $\begin{array}{l}\text { Spherical Equivalent }(\mathrm{SE} \text {, degree; mean } \pm \mathrm{SD} \text { ) } \\
\text { High myopia (no./\%) } \\
\text { History of previous non-vitreoretinal surgery } \\
\text { (no./\%) }\end{array}$ & $\begin{array}{c}-0.88 \pm 2.28 \\
0(0) \\
2(20)\end{array}$ & $\begin{array}{c}-2.14 \pm 2.76 \\
2(9.1) \\
2(9.1)\end{array}$ & $\begin{array}{l}0.21 \\
1.00 \\
0.57\end{array}$ \\
\hline $\begin{array}{l}\text { Follow-up time (months; mean } \pm \text { SD) } \\
\text { Pre-operative MH size }(\mu \mathrm{m} ; \text { mean } \pm \text { SD) } \\
\text { Pre-operative base of } \mathrm{MH}(\mu \mathrm{m} \text {; mean } \pm \mathrm{SD}) \\
\text { Pre-operative height of } \mathrm{MH}(\mu \mathrm{m} \text {; mean } \pm \mathrm{SD}) \\
\text { Postoperative MH closure (no./\%) } \\
\text { Postoperative IS/OS junction defect (no./\%) }\end{array}$ & $\begin{aligned} 24.45 & \pm 16.08 \\
296.68 & \pm 156.43 \\
2098.04 & \pm 2346.20 \\
633.83 & \pm 295.50 \\
10 & (100) \\
8 & (80)\end{aligned}$ & $\begin{array}{c}22.71 \pm 16.45 \\
257.56 \pm 137.72 \\
603.2 \pm 298.74 \\
353.72 \pm 235.97 \\
22(100) \\
7(31.8)\end{array}$ & $\begin{array}{c}0.88 \\
0.34 \\
0.004 \\
0.001 \\
1.00 \\
0.02\end{array}$ \\
\hline $\begin{array}{l}\text { BCVA (logMAR; mean } \pm S D) \\
\text { Preoperatively } \\
\text { Postoperatively } \\
\text { Change in BCVA }\end{array}$ & $\begin{array}{c}1.0 \pm 0.48 \\
0.64 \pm 0.51 \\
-0.36 \pm 0.34\end{array}$ & $\begin{array}{l}1.14 \pm 0.40 \\
0.46 \pm 0.21 \\
-0.69 \pm 0.31\end{array}$ & $\begin{array}{c}0.43 \\
0.30 \\
0.002\end{array}$ \\
\hline
\end{tabular}

Abbreviations: BCVA, best-corrected visual acuity; FTMH, full-thickness macular hole; IS/OS junction, inner segment/outer segment junction; MH, macular hole. ${ }^{a}$ Vertical traction $=$ The actual traction angle more than the critical vitreomacular traction angle (CVMTA) (27.2 degrees). ${ }^{\text {b Tangential }}$ traction $=$ The actual traction angle less than CVMTA.

operative and postoperative BCVA showed no difference between group B and control B individually, whereas the postoperative improvement in BCVA was less in group B $(P=0.002$; Table 2).

The measurement and calculation of the actual CVMTA is complicated; therefore, we directly measured the CVMTA on the computer monitor or on printouts without making adjustments for the magnification factors of the different OCT machines. By using line drawings and measurements, the actual CVMTA was found to be equivalent to $52^{\circ}$ on the machines' computer monitors or printouts on Optovue OCT; $45.6^{\circ}$ on the Zeiss OCT; and $52^{\circ}$ on the Heidelberg OCT. For practical purposes, we selected $45^{\circ}$ as the 'directly 
measured critical angle.' We subsequently examined whether this angle can be used to replace the actual CVMTA for determining anatomical changes and functional results. By using an angle of $45^{\circ}$ in all standard OCT images on the machines' monitors as the major criteria, we regrouped the IMH and FTMH cases into vertical and horizontal traction subgroups and reanalyzed the data. The results revealed that among the patients with IMHs, two with horizontal traction defined by the actual CVMTA were regrouped into the vertical traction subgroup (sensitivity $=100 \%$ and specificity $=85.7 \%$ ). Furthermore, among the patients with FTMHs, two with horizontal traction defined by the actual CVMTA were regrouped into the vertical traction group (sensitivity $=100 \%$ and specificity $=90.9 \%$ ). No patients with actual vertical traction were misgrouped into the horizontal traction subgroup. Comparisons between the patients with vertical and horizontal traction performed after regrouping based on the 'directly measured critical angle' yielded results similar to those obtained after grouping based on the actual CVMTA (data not shown).

\section{Discussion}

The actual vectors of forces acting on the macula in VMT are complex and difficult to calculate. The angle that the $\mathrm{PH}$ makes with the retina does not necessarily define the vector of forces active on the retina. Nonetheless, this attachment angle is the major index on an OCT image that may suggest the VMT direction. Theodossiadis et al studied traction direction and different foveal structural changes in IMHs and found that V-shaped traction (in the AP direction) tended to induce foveal detachment, whereas U-shaped traction (horizontally directed) was more likely to cause inner cystic changes. ${ }^{17}$ An important issue with that study is that the direction of vitreofoveal traction shown on the obtained OCT images did not represent the true TA, and the length of vitreofoveal adhesion used to define a V or U shape was not specified. Notably, our study revealed a greater length of VM attachment in the vertical traction group. We provide a method to evaluate the direction of VMT by measuring the pixels of an OCT computer image. VMT was known to be a dynamic process, and the TA changed over time. Thus, we selected OCT images captured preoperatively or after 6 months of follow-up without configuration changes for evaluation and measurement. We used horizontal and vertical scans for measurement because they are the standard scanning pattern for the three OCT machines and were convenient to use, although we understand that analyses utilizing more scanning angles may yield more reliable data. We calculated the true TA by considering the magnification factors in the vertical and horizontal directions of an OCT image and selected the largest angle from the four vitreofoveal traction lines in the two OCT images as the TA for a specific case. By using a regression model, we determined that a TA exceeding $27.2^{\circ}$ was associated with foveal detachment in an IMH case. Using this angle as a major parameter, in addition to the absence of the traction membrane bending toward the retinal surface, we identified the cases of significant AP vitreofoveal traction. In our case series, all eight cases in the vertical traction group presented with foveal detachment, whereas none of the 15 cases in the horizontal traction group presented foveal detachment. This high specificity and sensitivity consequently support our hypothesis that foveal detachment indicates significant AP VMT. If directly measured on the OCT monitors or printouts, this critical TA was equivalent to $52^{\circ}$ for OCT performed using Optovue and Heidelberg HRA machines, and $45.6^{\circ}$ for that performed using the Zeiss machine. For clinical convenience, we selected $45^{\circ}$ of oblique traction in an OCT image in all OCT machines to represent the directly measured critical angle on the machine monitors or printouts. By using this easily identifiable traction line as the major criterion, only two patients with horizontal traction defined by the actual critical angle in either the IMH or FTMH group were regrouped into the vertical traction subgroup (sensitivity $=100 \%$ for both groups and specificity $=85.7$ and $90.9 \%$ for the IMH and FTMH groups, respectively). No patients with actual vertical traction were misgrouped into the horizontal traction subgroup. This high sensitivity and specificity prompted us to suggest that in daily practice, $45^{\circ}$ can be used for direct measurements on machine monitors or printouts to distinguish the VMT direction and predict the morphological changes that will occur if IMHs develop.

The determination of the critical angle and identification of significant vertical traction have certain clinical implications. First, we found that vertical traction in an IMH induced higher retinal tissue elevation and a slightly wider impending hole base at the foveal site than did horizontal traction. These different structural changes indicated a more severe destruction of the inner and outer retina in the macular area, as evidenced by the trend toward worse BCVA in the vertical traction group. Second, our results indicated that vertical VMT resulted in a higher rate of FTMH development, and the duration between the IMH and FTMH stages was shorter in the vertical traction group. AP traction may sustain a stronger traction force on the fovea. Because the outer lamella has already been weakened by the detachment, once the inner roof is disrupted, FTMHs may rapidly develop.

In our study, the average length of VM attachment was $300 \pm 100.8 \mu \mathrm{m}$ in IMHs with horizontal traction, which was in concordance with the findings of Spaide et $a l^{19}$ 
However, we found that the average VM attachment was larger in IMHs with vertical traction $(593.9 \pm 142.3 \mu \mathrm{m}$; $P=0.0014)$; this length was in accordance with that obtained in the histological study by Kishi et $a l^{20} \mathrm{We}$ postulate that wider focal VM attachment suggests firmer adhesion. This feature may consequently delay vitreofoveal separation even when the VM separation outside the fovea has become significant. Thus, wider VM attachment tends to coexist with more vertical vitreofoveal traction. This higher VM separation eventually results in a higher chance of foveal detachment.

Our results revealed that similar to that in the IMH stage, group B had wide base separation and high foveal elevation in the early FTMH stage. However, the macular hole size did not significantly vary in the two groups. According to the conventional concept, the visual prognosis of FTMHs mainly depends on the macular hole size. ${ }^{21}$ However, in our study, although the postoperative macular hole closure rate was very high in both vertical and horizontal traction groups, the visual improvement was worse in the vertical traction group, despite the two groups having similar average hole sizes. The explanation for this may be that, as stated previously in this paper, early foveal detachment in the IMH stage may disturb the retinal OS and disrupt the photoreceptors at the foveal site. These injuries persist until the FTMH stage and result in less favorable outcomes after the macular hole is sealed. Furthermore, the wider gap at the outer retina in the vertical traction cases suggests more severe tissue loss or dehiscence in the vertical traction group. Conservative treatment is suggested for most IMHs. ${ }^{17,22-24}$ However, when the traction of the IMH is relatively vertical (in our study, exceeding $27.2^{\circ}$ ), the clinical course, initial BCVA, and BCVA outcomes are worse or tend to be worse than when the traction of the IMH is horizontal. The morphology displayed in OCT images can facilitate the differentiation of the traction vector; vertical traction in the early FTMH stage is a predictor of prolonged IS-OS defects and less favorable visual improvements after successful surgery.

In our study, under similar treatment criteria (ie, IMHs with a disturbed outer retina), group A showed a higher rate of FTMH development than did control A $(P=0.017$, data not shown). Furthermore, the incidence rate of an untreated IMH developing into an FTMH was higher than the general incidence rate previously reported. ${ }^{13,22}$ The average time required for an IMH to develop into an FTMH was shorter in cases of vertical traction than in those of horizontal traction. The cases that did not develop FTMHs were those wherein treatment with an intravitreal injection of $\mathrm{C}_{3} \mathrm{~F}_{8}$ was administered. Thus, early surgical intervention is suggested for IMHs with vertical traction.
In conclusion, traction direction may influence foveal structures and functional outcomes in idiopathic FTMHs. In this study, we first calculated the actual VMTA and further defined the CVMTA of vertical and horizontal traction. Compared with the patients with horizontal traction, those with vertical traction had prolonged IS-OS defects postoperatively (for IMHs and FTMHs), nearly worse pre-operative BCVA (for IMHs), and less BCVA improvement postoperatively (for FTMHs). For clinical use, we suggest using a TA of $45^{\circ}$ on computer monitors or printouts for the rapid screening of vertical traction. Early intervention may be considered in patients with IMHs with vertical traction to prevent FTMH development.

Summary

What was known before

- Vertical vitreomacular traction (VMT) is a common cause of idiopathic impending macular holes (IMHs) and fullthickness macular holes (FTMHs).

- The direction of traction angle (TA) may determine the early structural changes in the fovea.

What this study adds

- The actual angle separating vertical or horizontal VMT in IMHs is $27.2^{\circ}$, based on regression analysis of the TA and appearance of foveal detachment.

- IMHs or FTMHs with a vertical TA had a wider hole base and poorer clinical and visual prognosis than those with horizontal TA.

- For practical use, $45^{\circ}$ directly measured on screen monitors or printouts can be applied to differentiate vertical and horizontal traction in Optovue, Zeiss, and Heidelberg OCT machines.

\section{Conflict of interest}

The authors declare no conflict of interest.

\section{Acknowledgements}

We thank the National Taiwan University Hospital for supporting this study.

\section{References}

1 Johnson MW, Van Newkirk MR, Meyer KA. Perifoveal vitreous detachment is the primary pathogenic event in idiopathic macular hole formation. Arch Ophthalmol 2001; 119: 215-222.

2 Ezra E. Idiopathic full thickness macular hole: natural history and pathogenesis. Br J Ophthalmol 2001; 85: 102-109.

3 Kim JW, Freeman WR, El-Haig W, Maguire AM, Arevalo JF, Azen SP et al. Baseline characteristics, natural history, and risk factors to progression in eyes with stage 2 macular holes: 
results from a prospective randomized clinical trial. Ophthalmology 1995; 102: 1818-1829.

4 Smiddy WE, Flynn HW. Pathogenesis of macular holes and therapeutic implications. Am J Ophthalmol 2004; 137: 525-537.

5 Gass JDM. Idiopathic senile macular hole: its early stages and pathogenesis. Arch Ophthalmol 1988; 106: 629-639.

6 Duker JS, Kaiser PK, Binder S, De Smet MD, Gaudric A, Reichel E et al. The International Vitreomacular Traction Study Group classification of vitreomacular adhesion, traction, and macular hole. Ophthalmology 2013; 120: 2611-2619.

7 Asrani S, Zeimer R, Goldberg MF, Zou S. Serial optical sectioning of macular holes at different stages of development. Ophthalmology 1998; 105: 66-77.

8 Azzolini C, Patelli F, Brancato R. Correlation between optical coherence tomography data and biomicroscopic interpretation of idiopathic macular hole. Am J Ophthalmol 2001; 132: 348-355.

9 Haouchine B, Massin P, Gaudric A. Foveal pseudocyst as the first step in macular hole formation: a prospective study by optical coherence tomography. Ophthalmology 2001; 108: 15-22.

10 Kishi S, Kamei Y, Shimizu K. Tractional elevation of Henle's fiber layer in idiopathic macular holes. Am J Ophthalmol 1995; 120: 486-496.

11 Kishi S, Takahashi H. Three-dimensional observations of developing macular holes. Am J Ophthalmol 2000; 130: 65-75.

12 McDonnell PJ, Fine SL, Hillis AI. Clinical features of idiopathic macular cysts and holes. Am J Ophthalmol 1982; 93: 777-786.

13 Takahashi A, Nagaoka T, Yoshida A. Stage 1-A macular hole: a prospective spectral-domain optical coherence tomography study. Retina 2011; 31: 127-147.
14 Yeh PT, Chen TC, Yang CH, Ho TC, Chen MS, Huang JS et al. Formation of idiopathic macular hole-reappraisal. Graefes Arch Clin Exp Ophthalmol 2010; 248: 793-798.

15 Takahashi A, Nagaoka T, Ishiko S, Kameyama D, Yoshida A. Foveal anatomic changes in a progressing stage 1 macular hole documented by spectral-domain optical coherence tomography. Ophthalmology 2010; 117: 806-810.

16 Takahashi A, Yoshida A, Nagaoka T, Kagokawa H, Kato Y, Takamiya A et al. Macular hole formation in fellow eyes with a perifoveal posterior vitreous detachment of patients with a unilateral macular hole. Am J Ophthalmol 2011; 151: 981-989.

17 Theodossiadis G, Petrou P, Eleftheriadou M, Kagokawa S, Kato Y, Takamiya A et al. Focal vitreomacular traction: a prospective study of the evolution to macular hole: the mathematical approach. Eye 2014; 28: 1452-1460.

18 Gass JDM. Reappraisal of biomicroscopic classification of stages of development of a macular hole. Am J Ophthalmol 1995; 119: 752-759.

19 Spaide RF, Wong D, Fisher Y, Goldbaum M. Correlation of vitreous attachment and foveal deformation in early macular hole states. Am J Ophthalmol 2002; 133: 226-229.

20 Kishi S, Demaria C, Shimizu K. Vitreous cortex remnants at the fovea after spontaneous vitreous detachment. Int Ophthalmol 1986; 9: 253-260.

21 Ullrich S, Haritoglou C, Gass Ct, Schaumberger M, Ulbig W, Kampik A. Macular hole size as a prognostic factor in macular hole surgery. Br J Ophthalmol 2002; 86: 390-393.

22 de Bustros S. Group VfPoMHS. Vitrectomy for prevention of macular holes: results of a randomized multicenter clinical trial. Ophthalmology 1994; 101: 1055-1060.

23 Hikichi T, Yoshida A, Akiba J, Trempe CL. Natural outcomes of stage 1, 2, 3, and 4 idiopathic macular holes. Br J Ophthalmol 1995; 79: 517-520.

24 La Cour M, Friis J. Macular holes: classification, epidemiology, natural history and treatment. Acta Ophthalmol Scand 2002; 80: 579-587. 